\section{References}

1 C. L. Forgy, Artificial Intelligence, 19 (1982) 17-37.

2 F. Barachini, 8th International Workshop on Expert Systems \& their Applications, Avignon General Conference Vol. 2, EC2, Gerfau, Paris, 1988, pp. 217-234.

3 A. Gupta, 5th International Workshop on Expert Systems and their Applications, Avignon, EC2, Gerfau, Paris, 1985, pp. $26-57$.

4 M. R. Genesereth, Proceedings on the 3rd AAAI Conference, Kaufman, Los Altos, CA, 1983, pp. 119-124.

5 S. Russell, The Complete Guide to MRS, Report No. STANCS-85-1080, Stanford University, Stanford, June, 1985, 121 pp.
6 B. Silver, Studies in Computer Science and Artificial Intelligence, Vol. 1, North-Holland, Amsterdam, 1986.

7 J. Treur, Informatie, 30 (12) (1988) 909-972.

8 R. Davis and B. G. Buchanan, in B. G. Buchanan and E. H. Shortliffe (Editors), Rule-Based Expert Systems, Addison Wesley, Massachussetts, 1984, pp. 507-530.

9 M. L. Wright, M. W. Green, G. Fiegl and P. F. Cross, $A n E x$ pert System for Real-Time Control, IEEE Software, IEEE Computer Society, March, 1986, pp. 16-24.

M. De Winter, D. Grietens and Dr. M. Rijckaert are at the Chemical Engineering Department, KU Leuven, De Croylaan 46, 3030 Heverlee, Belgium.

\title{
An expert system for the validation of high- performance liquid chromatographic methods
}

\author{
L. M. C. Buydens and J. A. van Leeuwen \\ Nijmegen, The Netherlands

\section{Mulholland Cambridge, U.K.}

\section{B. G. M. Vandeginste and G. Kateman Nijmegen, The Netherlands}

A method validation expert system, developed as part of Esprit project ESCA, is described. The proper validation of high-performance liquid chromatography methods is an issue of rapidly growing importance. This is due to the increasing demands of good laboratory practice. Since validation involves a lot of statistical calculation and interpretation with which most analysts are not familiar, it is often neglected in the method development process. An expert system that provides this knowledge and experience is therefore very useful.

\section{Introduction}

Method validation is gaining rapidly in importance due to the increasing demands of good laboratory practice. This is especially true in the area of pharmaceutical analysis, where regulatory bodies pose increasing demands on the analytical methods. The Esprit project ESCA aims to build demonstrator expert systems for method development in high-performance liquid chromatography (HPLC) analysis of pharmaceutical compounds. The general approach of the project is described in the paper on
ESCA, elsewhere in this issue ${ }^{1}$. Such a system should include all necessary steps from the selection of initial HPLC conditions up to the validation of the method.

This resulted in four expert systems, each having a specific task covering the whole development process. The expert system for the selection of initial conditions provides chromatographic conditions that yield acceptable retention times for all peaks in the chromatogram. When a chromatogram is obtained in this way it may be necessary to optimise the selectivity to obtain an optimal distribution of the peaks over the chromatogram. This is done by the expert system for the selectivity optimization. The expert system for the optimization of chromatographic and instrumental parameters aims to obtain an acceptable resolution and signal-to-noise ratio in the shortest possible time. The task of the method validation expert system is to validate the HPLC method that has been selected and optimised in the other expert systems of ESCA. All these domains have been described elsewhere ${ }^{1,2}$. This paper focuses on the expert system for method validation. In the subsequent sections the scope and aproach in this expert system is described.

\section{Scope of the method validation expert system}

Method validation is a very broad concept. The full validation of a method comprises the testing of 
different aspects of its performance such as accuracy, precision, sensitivity, selectivity and limitations. The expert system described here concentrates on the precision testing of an HPLC method. Precision testing is the determination of the random error. Many methods will be applied under slightly different conditions from those of their development. The method may be used on other instruments, by other analysts or even in other laboratories. Therefore an estimation of the precision is a very important aspect in the method validation. According to Youden and Steiner ${ }^{3}$ one can distinguish a repeatability test and a reproducibility test. Repeatability testing involves testing the performance of a method when repeated under the same conditions, on the same instrument and by the same analyst. The reproducibility of a method is its precision under changing conditions and environments, e.g. on other instruments, by other analysts or in other laboratories. A reproducibility test hence involves an interlaboratory study which implies much effort and expense. The interlaboratory test of a method is doomed to failure when the method is not rugged for some slightly changing environmental and operational conditions that can be expected when the method is transferred to another site. Before organizing a large interlaboratory reproducibility test, the method can be intralaboratory tested for its ruggedness with respect to expected changes.

The evaluation of the precision often involves the use of more or less complex statistics. Most analysts therefore consider it a tedious task to test the precision of a developed method. An expert system that proposes the experiments that should be carried out, performs the appropriate statistical tests and interprets the results, and subsequently providing advice on the respecification of the method if the test fails, would be very useful. The expert system that has been developed in the ESCA project provides such advice on the repeatability and the ruggedness testing of HPLC methods ${ }^{4,5}$.

In this article we focus on the ruggedness testing part of the expert system ${ }^{5}$.

\section{Ruggedness system}

The use of statistical experimental design has been proposed by Youden and Steiner ${ }^{3}$ to evaluate the ruggedness of a method for certain factors. More recently these designs have been applied in practice for the ruggedness testing of HPLC methods ${ }^{6-9}$.

The ruggedness expert system described here contains the necessary knowledge and experience for advising the user on the use and interpretation of appropriate statistics. The expert system basically consists of a separate module for each of the following four steps:

- method description;

- the selection of important factors;

- the selection of the experimental design;

- processing and interpreting the results.

\section{The method description module}

The user is asked to fully specify the method that will be tested. This includes information about several aspects of the analytical procedure, all of which may influence the degree and extent of the testing procedure. This module contains knowledge on the normal range of the specifications. If the system accepts the user's method description, the user can safely rely on the result of a further consultation. Only when the method description is very incomplete is some caution required. The conclusions may not be fully significant when too much information is missing. In all other cases the user can be confident about the results when the method description is accepted by the system.

The requested information is:

- information about the analytical procedure of the HPLC method, such as sample preparation, column, flow-rate, etc.

- chromatographic results, such as retention times and minimal resolution between two peaks.

- intended application area of the method. Different levels of validation are required, depending on whether the method is only used a few times or is applied for many samples in different laboratories. Also, before a method is submitted to regulatory bodies, a thorough precision test should be carried out.

- finally, knowledge about the availability of instruments is needed to build up the full validation procedure.

The method description module guides the user in providing all necessary information, without asking for superfluous or irrelevant data. It contains common sense knowledge about chromatographic practice and asks only information that applies to the specific case. The consultation of this module is the basis for the further steps in the ruggedness system.

\section{The factor selection module}

In this step all factors that are likely to vary in the daily use of the method and that are suspected to influence its performance are identified. Since HPLC is a rather complicated analytical technique, many parameters must be considered. The experienced analyst has however more or less strong ideas about the most important factors that are relevant to the behaviour of the system. For example, a method should be rugged for the temperature when it is clear 
from the method description that no temperature control is provided. When the method is to be used over a long period of time and/or in other laboratories, then it is clear that the temperature of the environment will vary. When the method is not rugged for these variations this can cause alterations in its behaviour and wrong results may be obtained. The factor selection module covers the knowledge about the significant selection of these factors. This involves experience about the suspected variations of the selected factors in different environments ${ }^{10}$.

Another feature of this module is that the user is allowed to add or delete factors that are chosen by the expert system. The levels of the selected factors can also be altered. This feature is provided to allow the user to introduce his/her knowledge about a specific practical situation. For good laboratory practice these alterations are registered so that they can always be traced afterwards. When the user agrees with the proposed set of factors, all information is stored and the system can continue.

\section{Module to select an experimental design}

The number of factors that may influence the method's performance, when applied in practice, is very large. Even when only the most important factors are selected a large number of experiments is still required. Experimental designs that allow as much information as possible to be extracted from a minimum number of experiments are necessary. When it can be assumed that there are no higher order interactions between the different factors, which is often a realistic assumption, then the fractional factorial designs are the most efficient for this purpose $^{3}$. The designs that are implemented in the expert system are:

- full factorial designs;

- half fractional factorial designs;

- saturated factorial designs; and

- reflected saturated factorial designs.

The actual choice of a particular design depends on the number of factors and levels that are selected by the factor selection module. When the effects of only two or three factors are to be established at two levels then the full fractional designs are selected, otherwise the saturated fractional designs are preferred. When the expert system has made a decision it presents the selected design together with the batch of experiments that are to be carried out. At this stage the user leaves the expert system to perform the required experiments.

\section{Module for processing and interpretation of results}

When the experimental design has been selected an appropriate spreadsheet is created where the re- sults of the experiments can be entered. When all data have been collected the expert system can start processing the results. First the relevant chromatographic results from each experiment are calculated. These include: concentrations, peak efficiency, resolutions etc. When these parameters are obtained the calculations can start to find the effects of the different factors ${ }^{11}$. The method is supposed to be repeatable before the ruggedness test is started. To check if this is valid over the whole experiment all standard errors are examined. Since the analyte concentrations are the most important chromatographic results, the main effects of all factors on the calculated concentrations are calculated first and compared with a prespecified tolerance level. If any factor shows an unacceptably large effect on the concentrations, the method fails on the ruggedness test. The main effects on all the other parameters, such as peak height and resolution, are then investigated in the same way. When the tolerance level for these parameters is exceeded, the ruggedness test does not fail, but warnings are flagged to the user. If the ruggedness test of a specified method fails, the user is advised to respecify the method and to repeat the ruggedness test. For obtaining meaningful advice on this issue, this expert system must be linked to the other expert systems that were developed within the ESCA project. The most straightforward is the integration with the expert system for the optimization of instrumental and chromatographic parameters. This expert system can give advice on how to change the method in order to obtain improvements, e.g. in resolution. Integration of these two expert systems is proceeding.

When the method passes the ruggedness test a ruggedness report is printed. This includes a set of system suitability criteria. These are the maximum and minimum values that are found for chromatographic parameters such as resolution and peak height. Whenever the method is applied, the analyst can then use these values to evaluate the chromatographic system regarding its suitability for the method.

\section{Implementation}

The method validation system is implemented in Goldworks, one of the selected expert system shells in the ESCA project. Since method validation is a very complex and broad area, of which only a part is implemented in the present system, it was necessary to build the expert system in a way that is easily extendable. We chose a modular approach. The two main modules that constitute precision testing are the repeatability and the ruggedness system. These modules can be consulted separately. Each of these 


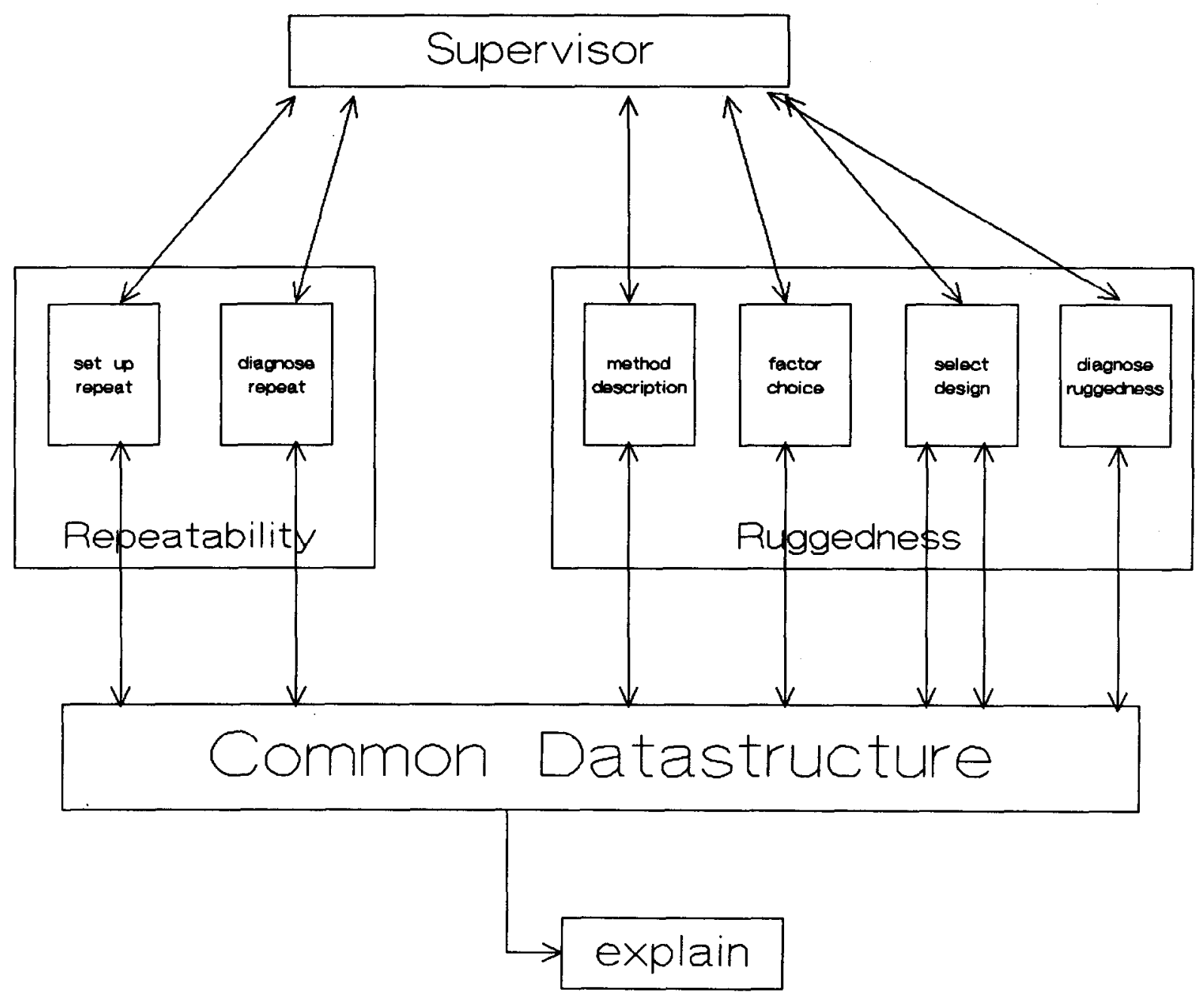

Fig. 1. Implementation architecture of the precision testing expert system.

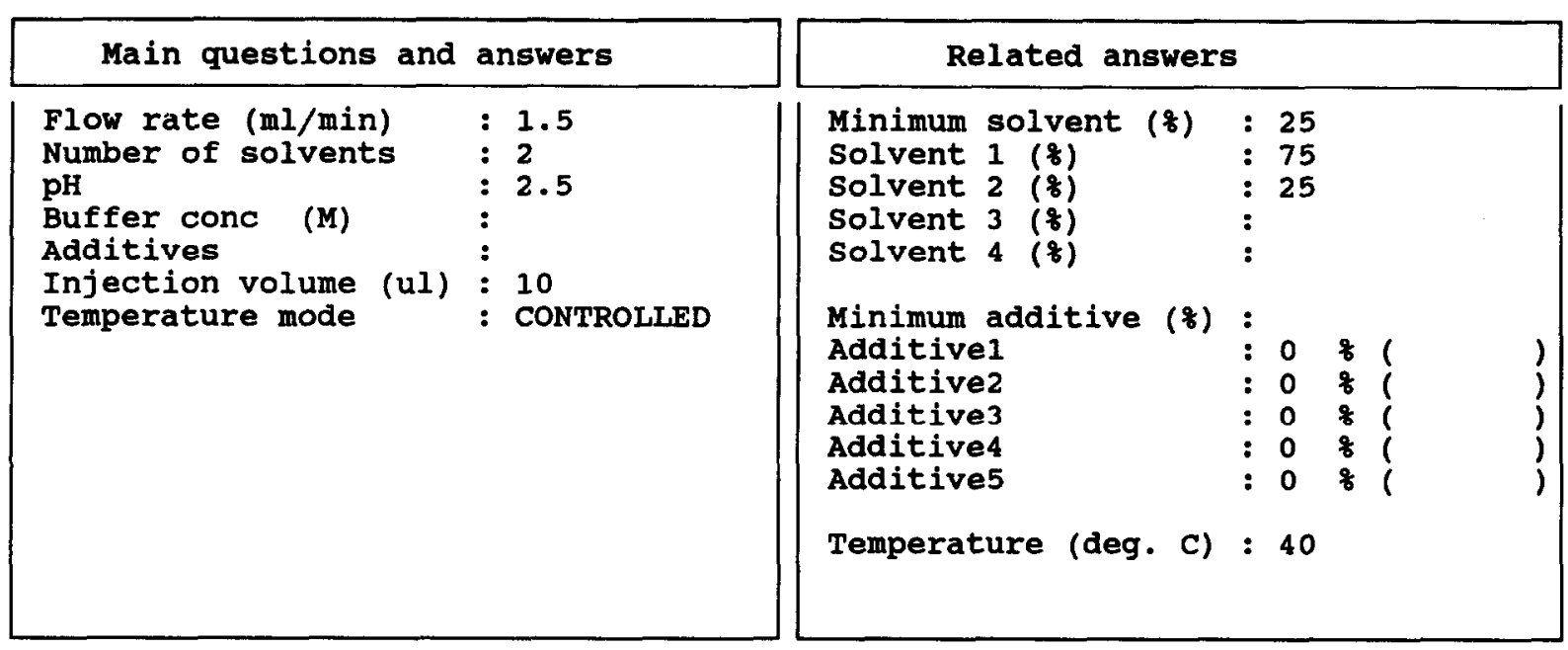

Fig. 2. Example of user interaction with the ruggedness expert system. 
main modules are in turn built from different submodules, and each submodule can also be consulted individually. A structure, called the supervisor, guides the user through the appropriate modules. It contains meta level or strategic knowledge on the next module to be consulted and therefore needs an overview of the results and data that are used in all separate modules. All data and results of the modules are stored in common database, accessible by all modules and the supervisor. The overall architecture is pictured in Fig. 1.

This architecture allows the integration of modules which contain very different types of knowledge. This is very useful since, as can already be seen from the ruggedness system modules, very different knowledge sources are necessary. Experiential as well as algorithmic knowledge sources must be combined in an integrated system to make it useful. This combination of modules containing different types of knowledge is a typical feature of second generation expert systems ${ }^{12}$.

In the ESCA project the stand-alone expert systems described elsewhere in this issue ${ }^{1}$ will be integrated. The architecture of the method validation expert system is flexible enough to allow an easy integration with the other expert systems.

Though user-friendliness was not the main concern in the project, attention was paid to developing an efficient and rather robust user interface. The questions are presented to the user in a window system. Explanations and additional help are provided where necessary. These can always be accessed by the user through special pop up windows. An example of the user interface is presented in Fig. 2.

\section{Conclusions}

The expert system for method precision testing, developed within the ESCA project, is a succesful stand-alone expert system. Validation of the system by means of 11 real test cases resulted in about $85 \%$ of success. Even for the cases where the conclusions were different from the real expert, the expert system choice was acceptable to the expert and in some cases even better than the real expert. A full description of the validation and further evaluation of the system is published elsewhere ${ }^{13}$. The implementation of the system allows future additions and integrations to be carried out with flexibility.

\section{Acknowledgement}

Part of this research is supported by the EEC as Esprit project P1570 Expert systems in chemical analysis (ESCA).

\section{References}

1 J. A. van Leeuwen, L. M. C. Buydens, B. G. M. Vandeginste and G. Kateman, Trends Anal. Chem., 9 (1990) 49.

2 D. Goulder, T. Blaffert, A. Blokland, L. M. C. Buydens, A. Chhabra, A. Cleland, N. Dunand, H. Hindriks, G. Kateman, J. A. van Leeuwen, D. L. Massart, M. Mulholland, G. Musch, P. Naish, A. Peeters, G. Postma, P. J. Schoenmakers, M. DeSmet and G. B. M. Vandeginste, Chromatographia, 26 (1988) 237-243.

$3 \mathrm{~W}$, J. Youden and E. H. Steiner, Statistical manual of the AOAC, AOAC, Washington, DC, 1975.

4 M. Mulholland, J. A. van Leeuwen and B. G. M. Vandeginste, Anal. Chim. Acta, 223 (1989) 183-192.

5 M. Mulholland, N. Dunand, A. Cleland, J. A. van Leeuwen and B. G. M. Vandeginste, J. Chromatogr., in press.

6 M. Mulholland and H. Waterhouse, J. Chromatogr., 395 (1987) 539-551.

7 M. Mulholland and J. Waterhouse, Chromatographia, 25 (9) (1988) 769-774.

8 M. Mulholland, P. J. Naish, D. R. Stout and J. Waterhouse, Chemometrics and Intelligent Laboratory Systems, 5 (1989) 262-270.

9 A. Mulholland, Trends Anal. Chem., 7 (1988) 383.

10 J. A. van Leeuwen, B. G. M. Vandeginste, G. Kateman, M. Mulholland and A. Cleland, submitted for publication.

11 G. Box, W. Hunter and J. Hunter, Statistics for Experiments, an Introduction to Design, Data Analysis and Model Building, Wiley, New York, 1978, pp. 291-453.

12 B. Maitre, T. Laasri, F. Mondot, F. Charpillet and J. P. Haton, Proceedings of the 9 th International Workshop on Expert Systems and their Applications, Specialised Conference on Second Generation Expert Systems, Avignon, May 29-June 2, 1989, EC2, Nauterre, 1989, pp. 237-251.

13 M. Mulholland, J. A. van Leeuwen and L. M. C. Buydens, J. Chromatogr., in press.

Drs. L. M. C. Buydens, J. A. van Leeuwen and G. Kateman are at the Department of Analytical Chemistry, Catholic University of Nijmegen, Nijmegen, The Netherlands.

Dr. M. Mulholland is at Philips Scientific, Cambridge, K.U.

Dr. B. G. M. Vandeginste is at Unilever Research, Vlaardingen, The Netherlands.

\section{Computer Corner Contributions}

Contributions of between 400 and 900 words are welcome in the following categories: hardware, software, chemical applications, mathematical tools and interfacing. Please send your papers either to:

TrAC Computer Comer, D.L. Massart, Vrije Universiteit Brussel, Fakulteit der Geneeskunde en der Farmacie, Farmaceutische Scheikunde, Laarbeeklaan 103, B-1090 Brussels, Belgium.

or

TrAC Computer Comer, A.P. Wade, Department of Chemistry, University of British Columbia, 2036 Main Mall, Vancouver, B.C. Canada V6T 1Y6. 